\title{
Axiomatic generalizations of OWA operators
}

\author{
Anna Kolesárová, ${ }^{1}$ Radko Mesiar, ${ }^{2,3}$ Andrea Stupňanová ${ }^{2}$ \\ ${ }^{1}$ Institute IEAM, FCHFT, Slovak University of Technology, 81237 Bratislava, Slovak Republic \\ anna.kolesarova@stuba.sk \\ ${ }^{2}$ Department of Mathematics, FCE, Slovak University of Technology, 81005 Bratislava, Slovak Republic \\ radko.mesiar@stuba.sk, andrea.stupnanova@stuba.sk \\ ${ }^{3}$ University of Ostrava, IRAFM, 30. dubna 22, Ostrava, Czech Republic
}

\begin{abstract}
Axiomatic generalizations of OWA operators are introduces and discussed. First, OMA operators based on comonotone modularity are recalled. Then, several kinds of comonotone pseudoadditivity based OWA generalizations are characterized and exemplified. Some of already known OWA generalizations are thus seen from new points of view. In several cases an integral representation of generalized OWA operators is included.
\end{abstract}

Keywords: OWA operator, OMA operator, GOWA operator, copula, Choquet integral, comonotonicity.

\section{Introduction}

OWA (Ordered Weighted Average) operators were introduced by Yager in 1988 [31]. Though we will deal with aggregation functions $A:[0,1]^{n} \rightarrow$ $[0,1]$ (i. e., non-decreasing functions satisfying two boundary conditions $A(0, \ldots, 0)=0$ and $A(1, \ldots, 1)=1$, for more details see $[1,3,11])$, formally OWA operators can be defined on any real interval $I \subseteq \mathbb{R}$ as follows:

Consider a normed weighting vector $\mathbf{w}=$ $\left(w_{1}, \ldots, w_{n}\right) \in[0,1]^{n}, \sum_{i=1}^{n} w_{i}=1$ (observe that $\mathbf{w}$ can be seen as a discrete probability distribution); then the function $\mathrm{OWA}_{\mathrm{w}}: I^{n} \rightarrow I$ given by

$$
\mathrm{OWA}_{\mathbf{w}}(\mathbf{x})=\sum_{i=1}^{n} w_{i} x_{\sigma(i)}
$$

is called an OWA operator (linked to w), where for $\mathbf{x}=\left(x_{1}, \ldots, x_{n}\right) \in I^{n}$ the permutation $\sigma$ : $\{1, \ldots, n\} \rightarrow\{1, \ldots, n\}$ satisfies $x_{\sigma(1)} \geq \cdots \geq$ $x_{\sigma(n)}$. Note that $x_{\sigma(i)}=\mathrm{OS}_{n-i+1}(\mathbf{x})$ can be seen as the $(n-i+1)$-th order statistics of the sample $\left(x_{1}, \ldots, x_{n}\right)$, i. e., $x_{\sigma(1)}$ is the maximal value in $\left(x_{1}, \ldots, x_{n}\right)$, while $x_{\sigma(n)}$ is the minimal value in $\left(x_{1}, \ldots, x_{n}\right)$.

There are more than 500 papers dealing with OWA operators, for more information we recommend an exhaustive survey [8]. There are also several generalizations of OWA operators, such as GOWA [28], IOWA [30, 31], two-dimensional OWA [2], IGOWA [19] and others. Three different looks on generalizations of OWA operators (basic, integral and axiomatic) are presented and discussed in our recent paper [23]. The aim of this contribution is a deeper look on the axiomatic generalizations of OWA operators. Single axioms required for considered particular generalizations express expected properties of aggregation functions required for particular applications. Our choice of axioms cannot be exhaustive. Nevertheless, we hope our choice of single axiomatic frameworks to be sufficiently representative.

The paper is organized as follows. In the next section, axiomatic characterization of OWA operators is recalled and generalized into OMA operators. In Section 3, comonotone pseudo-additive generalizations of OWA operators are treated, namely $\mathrm{V}$-OWA operators in Subsection 3.1, $\mathrm{V}$-OWA operators with special type of homogeneity in Subsection 3.2 and $\oplus$-OWA operators based on a generator $g$ in Subsection 3.3. Finally, some concluding remarks are added.

\section{Modular generalization of OWA operators}

Grabisch [10] has shown the link between OWA operators and the Choquet integral. This fact, together with the axiomatic characterization of the discrete Choquet integral due to Schmeidler [25], result into the axiomatic characterization of OWA operators acting on $[0,1]$. Indeed, OWA operators can be characterized as comonotone additive symmetric aggregation functions on $[0,1]$.

Recall that two vectors $\mathbf{x}, \mathbf{y} \in[0,1]^{n}$ are said to be comonotone whenever there is a common permutation $\sigma:\{1, \ldots, n\} \rightarrow\{1, \ldots, n\}$ such that $x_{\sigma(1)} \geq \cdots \geq x_{\sigma(n)}$ and $y_{\sigma(1)} \geq \cdots \geq y_{\sigma(n)}$. Moreover, an aggregation function $A:[0,1]^{n} \rightarrow[0,1]$ is comonotone additive whenever it satisfies

$$
A(\mathbf{x}+\mathbf{y})=A(\mathbf{x})+A(\mathbf{y})
$$

for all comonotone vectors $\mathbf{x}, \mathbf{y} \in[0,1]^{n}$ such that $\mathbf{x}+\mathbf{y} \in[0,1]^{n}$.

It can be shown that each OWA operator is idempotent, $\mathrm{OWA}_{\mathbf{w}}(c, \ldots, c)=c$ for each $c \in[0,1]$ and for any normed weighted vector $\mathbf{w}$, as well as positively homogeneous, $\mathrm{OWA}_{\mathbf{w}}(c \mathbf{x})=c \mathrm{OWA}_{\mathbf{w}}(\mathbf{x})$ for any $\mathbf{x} \in[0,1]^{n}$ and $c \geq 0$ such that also $c \mathbf{x} \in[0,1]^{n}$. 
The additivity property of aggregation functions can be generalized into the modularity. We say that an aggregation function $A:[0,1]^{n} \rightarrow[0,1]$ is modular whenever

$$
A(\mathbf{x} \vee \mathbf{y})+A(\mathbf{x} \wedge \mathbf{y})=A(\mathbf{x})+A(\mathbf{y})
$$

for any $\mathbf{x}, \mathbf{y} \in[0,1]^{n}$, where the lattice operations $\checkmark$ and $\wedge$ are defined as

$$
\mathbf{x} \vee \mathbf{y}=\left(\max \left(x_{1}, y_{1}\right), \ldots, \max \left(x_{n}, y_{n}\right)\right),
$$

and

$$
\mathbf{x} \wedge \mathbf{y}=\left(\min \left(x_{1}, y_{1}\right), \ldots, \min \left(x_{n}, y_{n}\right)\right) .
$$

Each modular aggregation function $A$ : $[0,1]^{n} \rightarrow[0,1]$ can be represented in the form

$$
A(\mathbf{x})=\sum_{i=1}^{n} f_{i}\left(x_{i}\right),
$$

where $f_{i}:[0,1] \rightarrow[0,1], i=1, \ldots, n$, is a nondecreasing function. If $A$ is also idempotent then $\sum_{i=1}^{n} f_{i}(x)=x$ for each $x \in[0,1]$, and hence each $f_{i}$ is 1- Lipschitz, $\left|f_{i}(x)-f_{i}(y)\right| \leq|x-y|$, and $f_{i}(0)=0$. This fact allows us to introduce an alternative axiomatic characterization of OWA operators.

Theorem 2.1 Let $A:[0,1]^{n} \rightarrow[0,1]$ be an aggregation function. Then it is an OWA operator if and only if it is comonotone modular, symmetric, and positively homogeneous.

The next generalization of OWA operators was proposed in [21].

Definition 2.2 Let $A$ be a symmetric idempotent comonotone modular aggregation function. Then $A$ is called OMA operator (Ordered Modular Average).

The next important result was shown in [21].

Theorem 2.3 Let $A:[0,1]^{n} \rightarrow[0,1]$ be a function. Then the following are equivalent:

1) $A$ is an OMA operator;

2) There are 1-Lipschitz non-decreasing functions $f_{1}, \ldots, f_{n}:[0,1] \rightarrow[0,1], \sum_{i=1}^{n} f_{i}(x)=x$ for each $x \in[0,1]$, and

$$
A(\mathbf{x})=\sum_{i=1}^{n} f_{i}\left(x_{\sigma(i)}\right) .
$$

3) There is a copula $C:[0,1]^{2} \rightarrow[0,1]$ and a symmetric capacity $m: 2^{\{1, \ldots, n\}} \rightarrow[0,1]$ such that

$$
\begin{aligned}
A(\mathbf{x}) & =\sum_{i=1}^{n}\left(C \left(x_{\sigma(i)}, m(\{\sigma(1), \ldots, \sigma(i)\})-\right.\right. \\
& -C\left(x_{\sigma(i)}, m(\{\sigma(1), \ldots, \sigma(i-1)\})\right),
\end{aligned}
$$

with convention $\{\sigma(1), \ldots, \sigma(i-1)\}=\emptyset$ if $i=1$.
For more details about copulas and symmetric capacities we recommend the monograph [11].

Example 2.4 For $n=2$, define $f_{1}, f_{2}:[0,1] \rightarrow$ $[0,1]$ by

$f_{1}(x)=\max \left(\frac{x}{3}, \frac{4 x-1}{6}\right), f_{2}(x)=\min \left(\frac{2 x}{3}, \frac{2 x+1}{6}\right)$.

Then the corresponding OMA operator $\mathrm{OMA}_{\left(f_{1}, f_{2}\right)}:[0,1]^{2} \rightarrow[0,1]$ is given by

$$
\mathrm{OMA}_{\left(f_{1}, f_{2}\right)}\left(x_{1}, x_{2}\right)=f_{1}\left(x_{\sigma(1)}\right)+f_{2}\left(x_{\sigma(2)}\right)
$$

and it is depicted in Figure 1.

Observe that

$$
\operatorname{OMA}_{\left(f_{1}, f_{2}\right)}\left|[0,0.5]^{2}=\operatorname{OWA}_{\left(\frac{1}{3}, \frac{2}{3}\right)}\right|[0,0.5]^{2}
$$

and

$$
\mathrm{OMA}_{\left(f_{1}, f_{2}\right)}\left|[0.5,1]^{2}=\mathrm{OWA}_{\left(\frac{2}{3}, \frac{1}{3}\right)}\right|[0.5,1]^{2} .
$$

Note that $\mathrm{OMA}_{\left(f_{1}, f_{2}\right)}$ can be seen as an ordinal sum of two OWA operators, namely of $\mathrm{OWA}_{\left(\frac{1}{3}, \frac{2}{3}\right)}$ acting on $[0,0.5]$ and of $\mathrm{OWA}_{\left(\frac{2}{3}, \frac{1}{3}\right)}$ acting on $[0.5,1]$, as proposed by De Baets and Mesiar in [5]. Moreover, $\mathrm{OMA}_{\left(f_{1}, f_{2}\right)}$ can be seen as a level dependent capacity $M$-based Choquet integral introduced by Greco et al. [12], with level dependent capacity $M:[0,1] \times 2^{\{1,2\}}$ given by

$$
M(t, E)=\left\{\begin{array}{ll}
m_{1}(E) & \text { if } t \in[0,0.5[ \\
m_{2}(E) & \text { otherwise }
\end{array} .\right.
$$

Here $m_{1}, m_{2}$ are symmetric capacities related to normed weighting vectors $\left(\frac{1}{3}, \frac{2}{3}\right)$ and $\left(\frac{2}{3}, \frac{1}{3}\right)$, respectively, i. e.,

$$
\begin{gathered}
m_{1}(\{1\})=m_{1}(\{2\})=\frac{1}{3} \text { and } m_{2}(\{1\})=m_{2}(\{2\})=\frac{2}{3}, \\
m_{1}(\emptyset)=m_{2}(\emptyset)=0 \text { and } m_{1}(\{1,2\})=m_{2}(\{1,2\})=1 .
\end{gathered}
$$

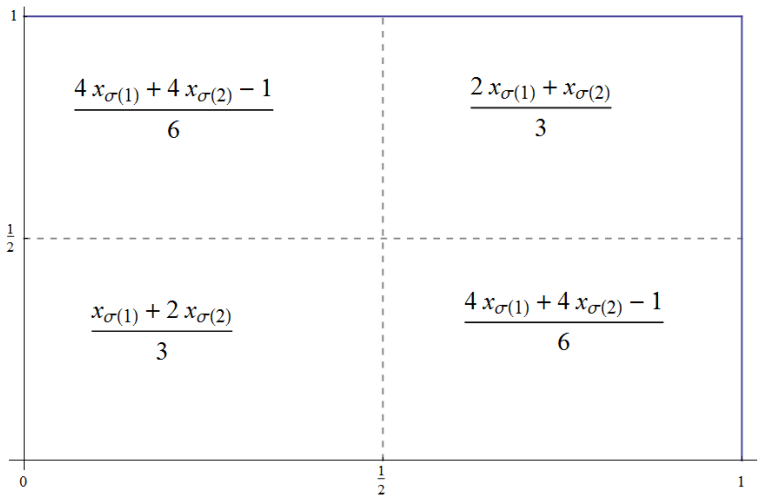

Figure 1: Formulae for $\mathrm{OMA}_{\left(f_{1}, f_{2}\right)}$ from Example 2.4.

As already mentioned, OMA operators can be seen as discrete copula-based integrals with respect to symmetric capacities, see (5). In this example, 
OMA operator depicted in Figure 1 is related to a symmetric capacity $m: 2^{\{1,2\}} \rightarrow[0,1]$ related to a normed weighting vector $\left(\frac{1}{2}, \frac{1}{2}\right)$, and the corresponding copula $C$ satisfies $C\left(x, \frac{1}{2}\right)=f_{1}(x), x \in$ $[0,1]$. As an example of such copula, exploiting method introduced in [13], one can consider a copula $C:[0,1]^{2} \rightarrow[0,1]$ given by

$$
C(x, y)= \begin{cases}\frac{2 x y}{3} & \text { if }(x, y) \in\left[0, \frac{1}{2}\right]^{2} \\ \frac{x+y-1+2 x y}{3} & \text { if }(x, y) \in\left[\frac{1}{2}, 1\right]^{2} \\ \frac{4 x y-\min (x, y)}{3} & \text { otherwise }\end{cases}
$$

\section{Pseudo-additive generalizations of OWA operators}

In this section we propose and discuss an axiomatic generalization of OWA's based on the idea to replace the additivity by pseudo-additivity. It is well known that an operation $\oplus:[0, \infty]^{2} \rightarrow[0, \infty]$ is called a pseudo-addition whenever it is monotone, symmetric, associative, continuous and 0 is its neutral element. For more details concerning the pseudo-additions we recommend [15, 24]. Each pseudo-addition $\oplus$ can be represented as an ordinal sum of generated pseudo-additions, $\oplus=$ $\left(\left\langle a_{k}, b_{k}, \varphi_{k}\right\rangle \mid k \in \mathcal{K}\right)$, where \{]$a_{k}, b_{k}[\mid k \in \mathcal{K}\}$ is a disjoint system of open subintervals of $[0, \infty]$, and $\varphi_{k}:\left[a_{k}, b_{k}\right] \rightarrow[0, \infty], k \in \mathcal{K}$, are continuous strictly monotone functions satisfying $\varphi_{k}\left(a_{k}\right)=0$. Then

$x \oplus y=\left\{\begin{array}{c}\varphi_{k}^{-1}\left(\min \left(\varphi_{k}\left(b_{k}\right), \varphi_{k}(x)+\varphi_{k}(y)\right)\right) \\ \quad \text { if }(x, y) \in] a_{k}, b_{k}\left[^{2} \text { for some } k \in \mathcal{K} .\right. \\ \max (x, y) \text { otherwise }\end{array}\right.$

Definition 3.1 Let $\oplus:[0, \infty]^{2} \rightarrow[0, \infty]$ be a given pseudo-addition, $\oplus=\left(\left\langle a_{k}, b_{k}, \varphi_{k}\right\rangle \mid k \in \mathcal{K}\right)$. An idempotent symmetric aggregation function $A$ : $[0,1]^{2} \rightarrow[0,1]$ is called a $\oplus$-OWA operator whenever it is comonotone pseudo-additive, $i$. e., if for any comonotone pair $\mathbf{x}, \mathbf{y} \in[0,1]^{n}$ such that $\mathbf{x} \oplus \mathbf{y}=\left(x_{1} \oplus y_{1}, \ldots, x_{n} \oplus y_{n}\right) \in[0,1]^{n}$ it holds

$$
A(\mathbf{x} \oplus \mathbf{y})=A(\mathbf{x}) \oplus A(\mathbf{y})
$$

\subsection{Idempotent pseudo-addition}

The only idempotent pseudo-addition is the standard maximum $\vee:[0, \infty]^{2} \rightarrow[0, \infty]$ given by $x \vee y=\max (x, y)$. Note that it corresponds to the empty ordinal sum, i.e., $\mathcal{K}=\emptyset$. The corresponding $\vee$-OWA operators are then characterized by the comonotone maxitivity and the symmetry. Recall that comonotone maxitive aggregation functions were studied in [22] and characterized as Sugeno integrals with respect to level dependent capacities. We have the next characterization of $\vee$-OWA operators.

Theorem 3.2 Let $A:[0,1]^{n} \rightarrow[0,1]$ be a function. Then the following are equivalent:
1) $A$ is a $\vee$-OWA operator;

2) There are increasing functions $f_{1}, \ldots, f_{n}$ : $[0,1] \rightarrow[0,1]$ such that

$$
\max \left(f_{1}(x), \ldots, f_{n}(x)\right)=x
$$

for each $x \in[0,1]$, and

$$
A(\mathbf{x})=\max \left(f_{1}\left(x_{\sigma(1)}\right), \ldots, f_{n}\left(x_{\sigma(n)}\right)\right) ;
$$

3) There is a level dependent symmetric capacity $M:[0,1] \times 2^{\{1, \ldots, n\}} \rightarrow[0,1]$ such that

$$
A(\mathbf{x})=\bigvee_{t \in[0,1]}\left(t \wedge M\left(t,\left\{i \in\{1, \ldots, n\} \mid x_{i} \geq t\right\}\right)\right) .
$$

For more details about level dependent capacities we recommend [14].

Obviously, Theorem 3.2 holds also for any pseudo-addition $\oplus$ such that it coincides with $\vee$ on $[0,1]^{2}$, i.e., $x \oplus y=\max (x, y)$ for each $x, y \in[0,1]$.

Example 3.3 For $n=2$ and $k \in] 0,1]$, consider $f_{1}, f_{2}:[0,1] \rightarrow[0,1]$ given by

$f_{1}(x)=\min (x, k), \quad f_{2}(x)= \begin{cases}0 & \text { if } x \leq k \\ x & \text { otherwise }\end{cases}$

Then the $\vee$-OWA operator $A:[0,1]^{2} \rightarrow[0,1]$ given by formula (7) is just $k$-median (idempotent nullnorm with annihilator $k$ ), see $[9,11]$,

$$
A\left(x_{1}, x_{2}\right)=\operatorname{med}\left(x_{1}, k, x_{2}\right) .
$$

Define a level dependent capacity $M:[0,1] \times$ $2^{\{1,2\}} \rightarrow[0,1]$ by

$$
\begin{aligned}
& M(t, \emptyset)=0, M(t,\{1,2\})=1, \\
& M(t,\{1\})=M(t,\{2\})=\left\{\begin{array}{ll}
1 & \text { if } t \leq k \\
0 & \text { otherwise }
\end{array} .\right.
\end{aligned}
$$

Then $M$ is symmetric and applying formula (8) we have an equivalent definition of $k$-median, i. e., an idempotent nullnorm with annihilator $a=k$,

$$
A\left(x_{1}, x_{2}\right)=\bigvee_{t \in[0,1]}\left(t \wedge M\left(t,\left\{i \in\{1,2\} \mid x_{i} \geq t\right\}\right)\right) .
$$

Obviously, if $\max \left(x_{1}, x_{2}\right)<k$ then

$$
M\left(\max \left(x_{1}, x_{2}\right),\left\{i \in\{1,2\} \mid x_{i} \geq \max \left(x_{1}, x_{2}\right)\right\}\right)=1
$$

while $M\left(t,\left\{i \in\{1,2\} \mid x_{i} \geq t\right\}\right)=0$ for each $t>$ $\max \left(x_{1}, x_{2}\right)$, and hence $A\left(x_{1}, x_{2}\right)=\max \left(x_{1}, x_{2}\right)$. Similarly, analyzing the remaining cases, we obtain

$$
A\left(x_{1}, x_{2}\right)= \begin{cases}\max \left(x_{1}, x_{2}\right) & \text { if } x_{1}, x_{2} \in[0, k[ \\ \min \left(x_{1}, x_{2}\right) & \text { if } \left.\left.x_{1}, x_{2} \in\right] k, 1\right] \\ k & \text { otherwise }\end{cases}
$$

Observe that $A$ is associative. 


\section{2. $\otimes$-homogeneous $\vee$-OWA operators}

Consider a pseudo-multiplication $\otimes:[0,1]^{2} \rightarrow[0,1]$ which possesses a neutral element 1, i. e., $x \otimes 1=$ $1 \otimes x=x$ for any $x \in[0,1]$, and which is monotone in each coordinate. Recall that this special aggregation functions is often called a semicopula [6]. Based on results of [21], we know that a comonotone maxitive aggregation function $A:[0,1]^{n} \rightarrow[0,1]$ which is positively $\otimes$-homogeneous, i. e., $A(c \otimes \mathbf{x})=c \otimes A(\mathbf{x})$ for each $c \in[0,1]$ and $\mathbf{x} \in[0,1]^{n}$, with $c \otimes \mathbf{x}=$ $\left(c \otimes x_{1}, \ldots, c \otimes x_{n}\right)$, is, in fact, the smallest universal integral on $[0,1]$ based on $\otimes$, see [16], with respect to some symmetric capacity $m: 2^{\{1, \ldots, n\}} \rightarrow[0,1]$. We have also a more transparent representation of these special $\vee$-OWA operators.

Theorem 3.4 Let $A:[0,1]^{n} \rightarrow[0,1]$ be a function and let $\otimes:[0,1]^{2} \rightarrow[0,1]$ be a semicopula. Then the following are equivalent:

1) $A$ is a positively $\otimes$-homogeneous $\vee$-OWA operator;

2) There is a cumulative normed weighting vector $\mathbf{v} \in[0,1]^{n}, i . e ., \mathbf{v}=\left(v_{1}, \ldots, v_{n}\right)$ and $0=v_{1} \leq v_{2} \leq \cdots \leq v_{n} \leq 1$, so that

$$
A(\mathbf{x})=\bigvee_{i=1}^{n}\left(v_{i} \otimes x_{\sigma(i)}\right) .
$$

Note that if $\otimes=$ min then $\vee-O W A$ operator given by $(9)$, i. e., $A(\mathbf{x})=\bigvee_{i=1}^{n}\left(v_{i} \wedge x_{\sigma(i)}\right)$, is the WOMax (Weighted Ordered Maximum) operators discussed already in [7] and it is related to the Sugeno integral [27].

If $\otimes$ is the standard product, $A(\mathbf{x})=\bigvee_{i=1}^{n}\left(v_{i} \cdot x_{\sigma(i)}\right)$, the link to the Shilkret integral [26] can be found. Similarly, when $\otimes=T$ is a strict t-norm, $A(\mathbf{x})=\bigvee_{i=1}^{n} T\left(v_{i}, x_{\sigma(i)}\right)$, an integral proposed by Weber [29] can be considered.

\subsection{Generated pseudo-addition}

Generated pseudo-additions are related to automorphism $g:[0, \infty] \rightarrow[0, \infty]$, and then $x \oplus y=$ $g^{-1}(g(x)+g(y))$, i.e., $\oplus=(\langle 0, \infty, g\rangle)$. In this case, the next result hold.

Theorem 3.5 Let $A:[0,1]^{n} \rightarrow[0,1]$ be a function and $\oplus=(\langle 0, \infty, g\rangle)$ be a generated pseudo-addition. Then the following are equivalent:

1) $A$ is a $\oplus$-OWA operator;

2) There is an OWA operator $B:\left[0, \infty\left[^{n} \rightarrow[0, \infty[\right.\right.$ so that

$$
A(\mathbf{x})=g^{-1}\left(B\left(g\left(x_{1}\right), \ldots, g\left(x_{n}\right)\right)\right) ;
$$

3) There is a normed weighting vector $\mathbf{w} \in[0,1]^{n}$ so that

$$
A(\mathbf{x})=\bigoplus_{i=1}^{n} g^{-1}\left(w_{i}\right) \otimes x_{\sigma(i)},
$$

where the pseudo-multiplication $\otimes:[0, \infty]^{2} \rightarrow$ $[0,1]$ is given by $x \otimes y=g^{-1}(g(x) \cdot g(y))$.

Note that once $\oplus$-OWA operator $A$ : $[0,1]^{n} \rightarrow[0,1]$ is known, the corresponding weights $w_{1}, \ldots, w_{n}$ can be determined as follows:

$$
\begin{gathered}
w_{1}=\frac{g(A(1,0, \ldots, 0))}{g(1)}, \\
w_{2}=\frac{g(A(1,1,0, \ldots, 0))-g(A(1,0, \ldots, 0))}{g(1)}, \\
\vdots \\
w_{n}=\frac{g(A(1, \ldots, 1)))-g(A(1, \ldots, 1,0))}{g(1)} .
\end{gathered}
$$

It is not difficult to check that each $\oplus$-OWA operator $A$ considered in Theorem 3.5 is positively $\otimes$ homogeneous, i. e., for any $\mathbf{x} \in[0,1]^{n}$ and $\left.c \in\right] 0, \infty[$ such that $\left(c \otimes x_{1}, \ldots, c \otimes x_{n}\right) \in[0,1]^{n}$ it holds $A(c \otimes \mathbf{x})=c \otimes A(\mathbf{x})$. Moreover, $A$ can be represented as a Choquet-like integral [20] with respect to a symmetric capacity $m: 2^{\{1, \ldots, n\}} \rightarrow[0,1]$,

$$
A(\mathbf{x})=(g-\mathbf{C h})-\int_{\{1, \ldots, n\}} \mathbf{x} \mathrm{d} m,
$$

where $m(E)=\sum_{i=1}^{\operatorname{card} E} w_{i}$.

As special examples one can consider GOWA operators [28]. For $\lambda>0$, consider $g(x)=x^{\lambda}$. Then the corresponding $\oplus$-OWA operator linked to a normed weighting vector $\mathbf{w}=\left(w_{1}, \ldots, w_{n}\right)$ is given by

$$
A(\mathbf{x})=\left(\sum_{i=1}^{n} w_{i} x_{\sigma(i)}^{\lambda}\right)^{\frac{1}{\lambda}}
$$

Observe that a dual aggregation function $A^{d}$ : $[0,1]^{n} \rightarrow[0,1]$ to an aggregation function $A$ is given by $A^{d}(\mathbf{x})=1-A\left(1-x_{1}, \ldots, 1-x_{n}\right)$. Then, if $A=\mathrm{OWA}_{\mathrm{w}}$, it is not difficult to check that also $A^{d}$ is an OWA operator, however, it is linked to the reversed weighting vector $\mathbf{w}^{\prime}=\left(w_{n}, \ldots, w_{1}\right)$, i. e., $A^{d}=\mathrm{OWA}_{\mathbf{w}^{\prime}}$.

Consider an automorphism $g:[0, \infty] \rightarrow[0, \infty]$ and a $\oplus$-OWA operator $A:[0,1]^{n} \rightarrow[0,1]$ linked to a normed weighting vector $\mathbf{w}$. Then

$$
A^{d}(\mathbf{x})=h^{-1}\left(\sum_{i=1}^{n} w_{n-i+1} h\left(x_{\sigma(i)}\right)\right),
$$

where $h:[0,1] \rightarrow[0, g(1)]$ is a decreasing bijection given by $h(x)=g(1-x)$. Observe that similar axiomatization of OWA operators can be done based on strict t-norms or t-conorms [15]. Consider, for example, a strict t-norm $T:[0,1]^{2} \rightarrow[0,1]$, i. e., $T(x, y)=\varphi^{-1}(\varphi(x)+\varphi(y))$ for some decreasing bijection $\varphi:[0,1] \rightarrow[0, \infty]$. Then a symmetric idempotent aggregation function $A:[0,1]^{n} \rightarrow[0,1]$ will be called a $T$-OWA operator whenever

$$
T(A(\mathbf{x}), A(\mathbf{y}))=A\left(T\left(x_{1}, y_{1}\right), \ldots, T\left(x_{n}, y_{n}\right)\right)
$$


for any comonotone vectors $\mathbf{x}, \mathbf{y} \in[0,1]^{n}$. Based on results of [4] the above constraint commuting problem has a solution

$$
A(\mathbf{x})=\varphi^{-1}\left(\sum_{i=1}^{n} w_{i} \varphi\left(x_{\sigma(i)}\right)\right) .
$$

Consider $T=\Pi$ the standard product. Then $\varphi$ is given by $\varphi(x)=-\log _{\lambda} x$, where $\lambda>1$ is a constant, and then (10) yields

$$
A(\mathbf{x})=\prod_{i=1}^{n} x_{\sigma(i)}^{w_{i}}
$$

i. e., each ПI-OWA operator is just the OWGA (Ordered Weighted Geometric Average) operator.

\section{Concluding remarks}

We have discussed several axiomatic generalizations of OWA operators. For some other generalizations we recommend [23]. Note that, following an idea of Einstein, an axiomatic characterization of any introduced and applied concept is the last theoretical step necessary for its deeper understanding, and an opening for further generalizations of the concerned concept. This is also the case of OWA operators, where their axiomatization has opened the door for several new kinds of aggregation functions, some of them being presented in this contribution. Based on the experience with applications of OWA operators, we expect the major applications of presented, axiomatically characterized aggregation functions in the area of multicriteria decision support and related fields.

Observe also that recently the OWA operators defined on lattices were proposed, see, e.g., [17, 18]. These operators were not yet axiomatically characterized, and thus this fact can be seen as an open problem. Moreover, it would be interesting to study axiomatically defined operators on lattices which could be seen as generalizations of OWA operators studied in $[17,18]$.

\section{Acknowledgments}

The support of the grants VEGA 1/0419/13 and VEGA $1 / 0420 / 15$ is kindly announced. Moreover, the work of R. Mesiar on this paper was supported by the European Regional Development Fund in IT4 Innovations Centre of Excellence project reg. no. CZ.1.05/1.1.00/02.0070.

\section{References}

[1] G. Beliakov, A. Pradera and T. Calvo, Aggregation Functions: a Guide for Practitioners. Springer, 2007.

[2] H. Bustince, T. Calvo, B. De Baets, J. Fodor, R. Mesiar, J. Montero, (...), A. Pradera, A class of aggregation functions encompassing two-dimensional OWA operators, Information Sciences, vol. 180, no. 10, pp. 1977-1989, 2010.

[3] T. Calvo, A. Kolesárová, M. Komorníková and R. Mesiar, Aggregation operators: properties, classes and construction methods, Aggregation Operators: New Trends and Applications, vol. 97, Physica-Verlag, Heidelberg, pp. 3-104, 2002.

[4] T. Calvo, R. Mesiar, Weighted triangular norms-based aggregation operators, Fuzzy Sets and Systems, vol. 137, pp. 3-10, 2003.

[5] B. De Baets, R. Mesiar, Ordinal sums of aggregation operators, Technologies for Constructing Intelligent Systems (B. Bouchon-Meunier, J. Gutiérrez-Ríos, L. Magdalena, and R. R. Yager, eds.), vol. 2: Tools, Physica-Verlag, Heidelberg, pp. 137-148, 2002.

[6] F. Durante, C. Sempi, Semicopulæ Kybernetika, vol. 43, no. 2, pp. 209-220, 2007.

[7] D. Dubois, H. Prade, A review of fuzzy set aggregation connectives, Inform.Sci., vol. 36, pp. 85-121, 1985.

[8] A. Emrouznejad, M. Marra, Ordered Weighted Averaging Operator: A Citation-Based Literature Survey, International Journal of Inteligent Systems, vol. 29, pp. 994-1014, 2014.

[9] J.C. Fodor, An extension of Fung?Fu's theorem, Int. J. Uncertainty, Fuzziness and Knowledge-Based Systems, vol. 4, pp. 235-243, 1996.

[10] M. Grabisch, Fuzzy integral in multicriteria decision making, Fuzzy Sets and Systems, vol. 69, no. 3, pp. 279-298, 1995.

[11] M. Grabisch, J.L. Marichal, R. Mesiar and E. Pap, Aggregation Functions. Cambridge University Press, New York, 2009.

[12] S. Greco, B. Matarazzo and S. Giove, The Choquet integral with respect to a level dependent capacity, Fuzzy Sets and Systems, vol. 175, no. 1, pp. 1-35, 2011.

[13] E.P. Klement, A. Kolesárová, R. Mesiar and C. Sempi, Copulas constructed from horizontal sections, Communications in Statistics Theory and Methods, vol. 36, no. 16, pp. 29012911, 2007.

[14] E.P. Klement, A. Kolesárová, R. Mesiar and A. Stupňanová, A generalization of universal integrals by means of level dependent capacities, Knowledge-Based Systems, vol. 38, pp. 14-18, 2013.

[15] E.P. Klement, R. Mesiar and E. Pap, Triangular Norms. Kluwer Academic Publishers, Dortrecht, 2000.

[16] E.P. Klement, R. Mesiar and E. Pap, A universal integral as common frame for Choquet and Sugeno integral, IEEE Transactions on Fuzzy Systems, vol. 18, pp. 178-187, 2010.

[17] I. Lizasoain, Quasi-OWA Operators on Complete Lattices. In H. Bustince et al. editors, $A g$ gregation Functions in Theory and in Practise, 
Advances in Intelligent Systems and Computing, proceedings of the 7th International Summer School on Aggregation Operators at the Public University of Navarra, vol. 228, pp. 521532, July 16-20, Pamplona, Spain, 2013.

[18] I. Lizasoain and C. Moreno, OWA operators defined on complete lattices, Fuzzy Sets and Systems, vol. 224, pp. 36-52, 2013.

[19] J.M. Merigó, A.M. Gil-Lafuente, The induced generalized OWA operator, Inform Sci., vol. 179, no. 6, pp. 729-741, 2009.

[20] R. Mesiar, Choquet-like Integrals, Journal of Mathematical Analysis and Applications, vol.194, no. 2, pp. 477-488, 2005.

[21] R. Mesiar, A. Mesiarová-Zemánková, The ordered modular averages, IEEE Transactions on Fuzzy Systems, vol. 19, no. 1, pp. 42-50, 2011.

[22] R. Mesiar, A. Mesiarová-Zemánková and K. Ahmad, Level-dependent Sugeno integral, IEEE Transactions on Fuzzy Systems, vol. 17, no. 1, pp. 167-172, 2009.

[23] R. Mesiar, A. Stupňanová and R. R. Yager, Generalizations of OWA operators, IEEE Transactions on Fuzzy Systems, 2015, in press, doi: 10.1109/TFUZZ.2015.2406888.

[24] P.S. Mostert, A.L. Shield, On the structure of semigroups on a compact manifold with boundary, Ann. of Math., vol. 65, pp. 117-143, 1957.

[25] D. Schmeidler, Integral representation without additivity, Proc. Amer. Math., vol. 97, no. 2, pp. 255-270, 1986.

[26] N. Shilkret, Maxitive measure and integration, Indag. Math., vol. 33, pp. 109-116, 1971.

[27] M. Sugeno, Theory of fuzzy integrals and its applications. $\mathrm{PhD}$ thesis, Tokyo Institute of Technology, 1974.

[28] G. Vitali, Sulla definizione di integraleoni di una variable, Ann. Mat. Pura Appl., vol. 2, no. 1, pp.111-121, 1925, English translation: On the definition of integral of functions of one variable, Rivista di Mathematica per le Scienze Sociali, vol. 20, pp. 159-168, 1977.

[29] S. Weber, Two integrals and some modified versions-Critical remarks, Fuzzy Sets and Systems, vol. 20, no. 1, pp. 97-105, 1986.

[30] R.R. Yager, Induced aggregation operators, Fuzzy Sets Systems, vol. 137, no. 1, pp. 59-69, 2003.

[31] R.R. Yager, On ordered weighted averaging aggregation operators in multicriteria decisionmaking, IEEE Trans Systems Man Cybernet, vol. 18, no. 1, pp. 183-190, 1988. 